\title{
Value of CT to predict surgically important bowel and/or mesenteric injury in blunt trauma: performance of a preliminary scoring system
}

\author{
Claire Faget $^{1}$ - Patrice Taourel ${ }^{1} \cdot$ Jonathan Charbit $^{3}$ - Alban Ruyer ${ }^{1}$. \\ Chakib Alili ${ }^{1} \cdot$ Nicolas Molinari $^{2} \cdot$ Ingrid Millet $^{1}$
}

\begin{abstract}
Objectives To evaluate the performance of a computed tomography (CT) diagnostic score to predict surgical treatment for blunt bowel and/or mesentery injury (BBMI) in consecutive abdominal trauma.

Methods This was a retrospective observational study of 805 consecutive abdominal traumas with 556 patients included and screened by an abdominal radiologist blinded to the patient outcome, to evaluate numerous CT findings and calculate their diagnostic performances. These CT findings were compared using univariate and multivariate analysis between patients who had a laparotomy-confirmed BBMI requiring surgical repair, and those without BBMI requiring surgery. A CT score was obtained with an internal bootstrap validation. Results Fifty-six patients $(10.1 \%)$ had BBMI requiring surgery. Nine CT signs were independently associated with BBMI requiring surgery and were used to develop a CT diagnostic score. The AUC of our model was 0.98 (95\% CI 0.96100 ), with a $\geq 5$ cut-off. Its diagnostic performance was determined by internal validation: sensitivity $91.1-100 \%$, specificity $85.7-97.6 \%$, positive predictive value $41.4-82.3 \%$ and negative predictive value 98.9-100\%. Bowel wall discontinuity and mesenteric pneumoperitoneum had the strongest
\end{abstract}

Ingrid Millet

i-millet@chu-montpellier.fr

1 Department of Medical Imaging, CHU Lapeyronie, 371 avenue Gaston Giraud, 34295 Montpellier, France

2 Department of Medical Information and Statistics, UMR 729 MISTEA, CHU Montpellier, Montpellier, France

3 Department of Intensive Care and Anesthesiology, CHU Lapeyronie, Montpellier, France association with BBMI requiring surgery $(\mathrm{OR}=128.9$ and 140.5 , respectively).

Conclusion We developed a reliable CT scoring system which is easy to implement and highly predictive of BBMI requiring surgery.

Key Points

- Finding of bowel wall discontinuity or mesenteric pneumoperitoneum indicates BBMI requiring surgery.

- Arterial mesenteric vessel extravasation requires surgery when in association with other CT findings.

- Our CT scoring system has excellent diagnostic performance in predicting BBMI requiring surgery.

Keywords MDCT · Bowel injury · Mesenteric injury · Blunt trauma $\cdot$ Scoring system

\section{Introduction}

Blunt bowel and mesenteric injuries (BBMI) occur in about $5 \%$ of blunt abdominal trauma patients [1]. Accurate and timely diagnosis is required because delays in diagnosis as short as $8-12 \mathrm{~h}$ after injury may increase the morbidity and mortality $[2,3]$. Identifying BBMI remains a challenge for trauma care providers as physical examination may be unrealistic in patients with multiple injuries, and it often takes hours before clinically apparent peritonitis signs and symptoms appear.

Multidetector computed tomography (MDCT) is the current accepted standard imaging modality for abdominal trauma [4]. It is highly reliable for intra-abdominal solid organ injury diagnosis and is now considered accurate in the diagnosis of bowel and mesenteric injuries complicating blunt abdominal trauma [5]. Many CT findings of BBMI have been reported, some of which are specific, but most are only 
suggestive, and the exact diagnostic performance of CT remains debatable, with experienced readers yielding more accurate diagnoses [2, 6-12]. Moreover, some BBMI, e.g. superficial bowel tear or mesenteric haematoma without bleeding, could sometimes be successfully managed with observational therapy. There is still controversy as to how reliably $\mathrm{CT}$ alone could help identify those BBMI requiring surgery. Lastly, some authors have underlined the high negative predictive value of CT for BBMI $[9,13,14]$, but the real predictive value of CT is unknown as, given the low incidence of BBMI, the studies were designed as case-control studies [9], or as surgical cohorts of patients treated by laparotomy for trauma $[10$, 13-15] but not in consecutive patients with abdominal trauma.

Our study was thus designed to assess the predictive value of CT signs for diagnosis of BBMI requiring surgery ("surgical BBMI") in consecutive patients with abdominal blunt trauma with the aim of developing and evaluating the performance of a CT diagnostic score for therapy planning.

\section{Materials and methods}

\section{Study population}

Institutional research ethics review board approval was obtained for this retrospective observational study and informed consent was waived.

The trauma database of the intensive care unit of our level 1 regional trauma centre was retrospectively reviewed to select all patients with an abbreviated injury score (AIS) for the abdomen-pelvis area of $\geq 1$ between April 2004 and December 2011. Among 805 patients who fulfilled this criterion, 249 were excluded for reasons detailed in Fig. 1. Finally, 556 consecutive patients were included, 409 male $(73.6 \%)$ and 147 female $(26.4 \%)$, with a median age of 29 years (interquartile range, IQR, $21-46$ years). The median injury severity score (ISS) was 14 (IQR 9-22).

\section{CT technique}

The CT studies were performed in our institution within $2 \mathrm{~h}$ of admission to the trauma centre $(n=521,93.7 \%)$, or in another institution before the patient was transferred to our trauma centre unit $(n=35,6.3 \%)$. All but 35 CTs were performed using a LightSpeed VCT 16- or 64-detector row scanner (GE Healthcare, Milwaukee, Wis), at $120 \mathrm{kVp}$, and the amperage setting ranged from 130 to $700 \mathrm{~mA}$, according to the body habitus. The CT images were reconstructed at $3-\mathrm{mm}$ section thickness in the axial, coronal and sagittal plane with native images available for interpretation. Intravenous contrast material (iohexol [Omnipaque 300], GE Healthcare; or iobitridol [Xénétix 350], Guerbet) was administrated at a rate of 3-4 mL/s via a power injector. The CT protocol at our institution included multi-phase acquisition with an arterial phase initiated using an automatic bolus-tracking program and a venous phase at a 70-80 s delay. A delayed sequence (3-5 min) was performed at the emergency radiologist's discretion. For the $35 \mathrm{CTs}$ that had been performed outside the institution, the arterial phase was only available in 8 patients $(22.9 \%)$, but venous and delayed phases were available in all cases. CT was performed without oral contrast in all cases.

\section{CT analysis}

All 556 CT examinations were retrospectively and independently reviewed by two radiologists (C.F., A.R.) who had respectively 6 and 4 years of experience in abdominal trauma imaging. Reviewers were blinded to the original radiology report, surgical findings and final outcome, but they were aware that the research study involved diagnosis of BBMI at CT.

The CT images were screened for a number of signs, as reported in Table 1. Haemoperitoneum was defined by highattenuation peritoneal fluid (35-60 HU) and was evaluated according to its amount, as proposed by Federle and Jeffrey [16]. Bowel enhancement was assessed subjectively by visual comparison and was classified as abnormal if it was decreased compared to the enhanced adjacent bowel loops. Bowel walls were considered thick if they were greater than $3 \mathrm{~mm}$ for the small bowel and $5 \mathrm{~mm}$ for the colon $[5,9,17]$. Bowel wall thickening was considered as focal if it was less than $10 \mathrm{~cm}$ in length, and non-focal if it was longer than $10 \mathrm{~cm}$. Diffuse bowel wall thickening with findings of hypovolaemic shock was not considered. Active arterial mesenteric vessel extravasation was considered if the extravasation appeared in the arterial phase and expanded at the later phases. When CT had not been performed with an arterial phase, it was considered when extravasation increased considerably (at least doubled) between the venous and delayed phases with coexistence of high and low density areas in the haemorrhage region, suggesting a high rate of active bleeding, as reported by Murakami et al. [18]. Sentinel clot sign was defined by focal high-density collection having an average $\mathrm{CT}$ density greater than $50 \mathrm{HU}$ [19]. Pneumoperitoneum was defined as "mesenteric" if the extra luminal air was seen only trapped in the mesentery, and as "free" if it was extended to the anterior part of the abdominal cavity, under the anterior parietal peritoneal layer. Mesenteric stranding was defined by a streaky soft-tissue infiltration of normal mesenteric fat. Beaded appearance of mesenteric vessels was defined as an irregular contour, and abrupt termination indicated by a lack of continuity or tapering of the artery or vein [9].

Injuries to solid visceral organs, bladder, large abdominal vessels, spine and pelvic bones were documented. Anterior abdominal wall injury was noted if there was stranding of 
Fig.1 Study flow chart

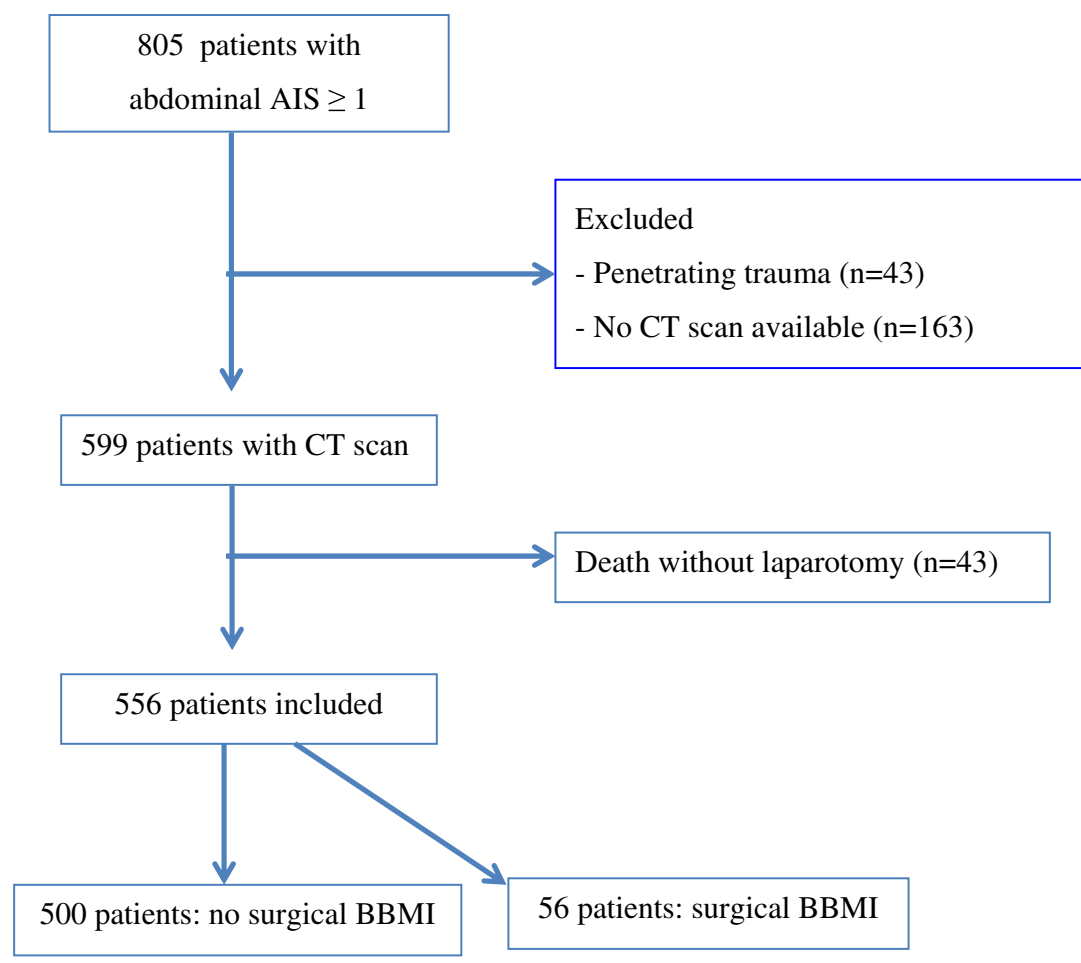

subcutaneous adipose tissue or if there was a rupture in the abdominal muscular wall or an anterior muscular haematoma.

\section{Reference standard}

Two of the authors (I.M. and J.C.) reviewed the trauma database information, official surgical notes and discharge summaries of all patients. Patient demographics, injury severity score and duration of hospitalization were obtained from the trauma registry.

The final diagnosis was established based on the surgical findings and pathology reports, which served as the reference standard for surgical BBMI. As proposed by Atri et al. [9], a surgical bowel injury was defined as a full-thickness

Table 1 CT signs evaluated

\begin{tabular}{ll}
\hline Mesenteric sign & Bowel sign \\
\hline $\begin{array}{l}\text { Haemoperitoneum } \\
\text { Mesenteric stranding }\end{array}$ & $\begin{array}{l}\text { Bowel wall discontinuity } \\
\text { Sentinel clot sign } \\
(=\text { mesenteric haematoma) }\end{array}$ \\
$\begin{array}{l}\text { Active arterial mesenteric } \\
\text { vessel extravasation }\end{array}$ & $\begin{array}{c}\text { Bowel wall thickening (site and } \\
\text { length) }\end{array}$ \\
$\begin{array}{c}\text { Irregular beading of the mesenteric } \\
\text { vessel }\end{array}$ & $\begin{array}{c}\text { Mesenteric pneumoperitoneum } \\
\text { enhancement }\end{array}$ \\
$\begin{array}{c}\text { Abrupt termination of the } \\
\text { mesenteric vessel }\end{array}$ & \\
\hline
\end{tabular}

perforation or seromuscular tear or devascularized bowel. A surgical mesenteric injury was defined as involving active mesenteric bleeding or mesenteric injury resulting in an ischemic bowel loop. Surgical BBMI required therapeutic laparotomy. Serosal tears of the bowel, bowel wall haematomas without tear, and mesenteric haematoma in the absence of active bleeding did not require surgical intervention and were thus considered as negative for surgical BBMI.

All patients referred to our trauma centre were admitted for at least $24 \mathrm{~h}$ of observation (more than 3 days for $95 \%$ of the total population). All patients were followed up for 2 months after discharge. Patients treated with non-operative management and discharged alive were thus considered as true negative for BBMI requiring surgery, as none of them returned for an occult BBMI.

\section{Statistical analysis}

Interobserver agreement for CT findings was determined with the $\mathrm{k}$ statistic and classified as follows: $\mathrm{k}=0-0.2$, slight agreement; $\kappa=0.21-0.4$, fair agreement; $\kappa=0.41-0.6$, moderate agreement; $\kappa=0.61-0.8$, substantial agreement; and $\mathrm{\kappa}=0.81-$ 1 , almost perfect agreement.

Disagreements between the two reviewers were resolved by consensus. Consensual data were then used for final statistics.

We compared epidemiological data and CT signs between patients with surgical BBMI and those without surgical BBMI. Pearson Chi square or Fisher tests were used for 
comparison of categorical variables, and Student $t$ test or Wilcoxon rank-sum test for comparison of continuous variables as appropriate. The diagnostic performance of each CT finding was also calculated.

All CT findings with a univariate $p$ value $\leq 0.1$ were entered into a multivariate logistic regression model to gauge their independent association with surgical BBMI. A stepwise procedure was used to select the final model. To establish a score, a rounded up numerical value was assigned to each of the significant variables included in the final prediction model, in relation to their $\beta$ parameter (logistic regression estimates). A receiver operating characteristic (ROC) curve was plotted to estimate the best cut-off of this model and its diagnostic performance. We used the bootstrap method to internally validate the score by sampling with replacement for 1000 iterations.

Statistical significance for all tests was set at $p<0.05$. Computer software (SAS, version 9.3, SAS Institute Cary, NC and $\mathrm{R}$, version 3.0.2, R Foundation for Statistical Computing) was used to perform the statistical analyses.

\section{Results}

\section{Study population}

There was no significant difference for age or sex ratio between patients with or without surgical BBMI [26.5 years (IQR 19-47.5) and 29 years (IQR 21-46), $p=0.36$; H/F 3.3 and 2.7, $p=0.56$, respectively]. The hospitalisation duration was significantly longer in the surgical BBMI group (median 21.5 days, IQR 11-32) vs. no surgical BBMI (median 14 days, IQR $8-25$ ) with $p=0.005$. The ISS was significantly higher in patients with surgical BBMI (18.5 vs. $14, p=0.0036)$. There were seven deaths, two of which involved surgical BBMI (one devascularized bowel and one seromuscular tear).

\section{Reference standard}

A total of 103 patients $(18.5 \%)$ underwent laparotomy, including 56 patients $(54.4 \%)$ with surgical BBMI. Eightyseven patients $(82.1 \%)$ were operated on within $24 \mathrm{~h}$ from the time of CT, including 50 patients $(57.5 \%, 50 / 87)$ with BBMI requiring surgery, whereas 16 patients were operated on $24 \mathrm{~h}$ after $\mathrm{CT}$, including 6 patients $(37.5 \%, 6 / 16)$ with BBMI requiring surgery.

Surgical findings for the 56 patients with BBMI requiring surgery were distributed as follows: 3 had mesenteric lesions which corresponded to active arterial bleeding, 24 bowel injuries including 17 bowel perforation, 6 seromuscular tears and 1 ischemic bowel wall; 29 had associated bowel and mesenteric injuries including 14 mesenteric bleeding, 20 bowel ischemia, 9 seromuscular tears and 10 bowel perforation. Among these 56 patients, 19 needed multiple surgical repairs and 37 single repairs, and treatments were as follows: 33 bowel resections (21 for ischemic bowel and 12 for multiples perforations), 30 bowel sutures and 17 surgical haemostasis for active bleeding. There was no BBMI requiring surgery in the 47 others patients who underwent laparotomy, and surgical treatments were as follows: 27 splenectomies, 5 nephrectomies, 5 hepatic repairs (suture), 8 hepatic packing and 2 diaphragmatic sutures.

The remaining patients, after excluding the five deaths $(n=$ $448,80.6 \%$ ), were discharged alive without abdominal surgical procedure during the hospitalization or surgery during follow-up.

\section{CT univariate analysis}

Tables 2 and 3 show results of univariate analysis, and diagnostic performance of each CT finding. All CT findings evaluated were significantly associated with an increased probability of surgical BBMI, except for length of bowel wall thickening $(p=0.22)$.

Bowel wall discontinuity was noted in 20 patients; there was only one false positive discontinuity in the colic bowel in a patient who had undergone laparotomy with no abdominal lesion and who died just after surgery because of nonabdominal haemorrhagic shock.

A free pneumoperitoneum was lacking in 12/27 (44.4\%) patients with a perforated bowel, whereas a mesenteric pneumoperitoneum was lacking in 19/27 (70.4 \%). In $8 / 27$ (29.6\%) patients with small bowel perforation, there was neither free nor mesenteric pneumoperitoneum.

There were six cases of false positives for arterial mesenteric extravasation. Among them, four underwent laparotomy without active mesenteric bleeding mentioned in the surgical report or without any haemostasis procedure, but two of them died after the surgical procedure due to haemorrhagic shock. The last two had active bleeding noted in the meso-sigmoid and the other in the small omentum, and both were conservatively treated favourably with spontaneous regression of bleeding.

Among the associated intra-abdominal lesions, only trauma of the spleen and of the anterior abdominal wall were significantly associated with surgical BBMI $(p=0.0077$ and $<0.0001$, respectively).

\section{Multivariate analysis}

Nine CT signs were independently associated with surgical BBMI, as listed in Table 4.

The ROC curve derived from our scoring system (Fig. 2) showed an AUC of 0.98 (0.96-1) with a cutoff $\geq 5$ for the best discriminative diagnostic performance. Using a cut-off $\geq 5$, our CT score had $96.4 \%$ sensitivity, $91.5 \%$ specificity, $56.2 \%$ positive predictive value 
Table 2 Univariate analysis of each CT finding in the general population and according to the presence of surgical BBMI

\begin{tabular}{|c|c|c|c|c|c|}
\hline & & $\begin{array}{l}\text { Overall } \\
N=556\end{array}$ & $\begin{array}{l}\text { Surgical BBMI } \\
N=56\end{array}$ & $\begin{array}{l}\text { No surgical BBMI } \\
N=500\end{array}$ & $p$ value \\
\hline \multirow[t]{3}{*}{ Haemoperitoneum } & None & $157(28.2)$ & $3(5.4)$ & $154(30.8)$ & \multirow[t]{3}{*}{$<0.0001$} \\
\hline & 1 (small amount) & $233(41.9)$ & $14(25)$ & $219(43.8)$ & \\
\hline & 2 (abundant) & $166(29.9)$ & $39(69.6)$ & $127(25.4)$ & \\
\hline Free pneumoperitoneum & & $21(3.8)$ & $17(30.4)$ & $4(0.8)$ & $4.3 \mathrm{E}-15^{\mathrm{b}}$ \\
\hline Mesenteric pneumoperitoneum & & $12(2.5)$ & $10(17.9)$ & $2(0.4)$ & $2.8 \mathrm{E}-09^{\mathrm{b}}$ \\
\hline \multirow[t]{2}{*}{ Bowel wall thickening } & None & $474(85.2)$ & $18(32.1)$ & $456(91.2)$ & \multirow[t]{2}{*}{$<0.0001$} \\
\hline & Present & $82(14.8)$ & $38(67.9)$ & $44(8.8)$ & \\
\hline \multirow[t]{2}{*}{ Bowel wall thickening length $(N=82)$} & Focal & $49(59.8)$ & $20(52.6)$ & $29(65.9)$ & \multirow[t]{2}{*}{0.22} \\
\hline & Non-focal & $33(40.2)$ & $18(47.4)$ & $15(34.1)$ & \\
\hline Arterial mesenteric vessel extravasation & a & $21(3.8)$ & $15(26.8)$ & $6(1.2)$ & $6 \mathrm{E}-12^{\mathrm{b}}$ \\
\hline Mesenteric haematoma & & $56(10.1)$ & $24(42.9)$ & $32(6.4)$ & $<0.0001$ \\
\hline Mesenteric stranding & & $85(15.3)$ & $33(58.9)$ & $52(10.4)$ & $<0.0001$ \\
\hline Abrupt termination of mesenteric vessel & a & $18(3.2)$ & $14(25)$ & $4(0.8)$ & $5.3 \mathrm{E}-12^{\mathrm{b}}$ \\
\hline Beaded mesenteric vessel & a & $13(2.3)$ & $7(12.5)$ & $6(1.2)$ & $8 \mathrm{E}-05^{\mathrm{b}}$ \\
\hline Decreased bowel wall enhancement & a & $33(6)$ & $22(39.3)$ & $11(2.2)$ & $1.6 \mathrm{E}-16^{\mathrm{b}}$ \\
\hline Bowel wall discontinuity & a & $20(3.6)$ & $19(33.9)$ & $1(0.2)$ & $9.8 \mathrm{E}-20^{\mathrm{b}}$ \\
\hline \multicolumn{6}{|l|}{ Associated abdominal injury } \\
\hline Liver & & $224(40.4)$ & $18(32.1)$ & $206(41.4)$ & 0.1824 \\
\hline Spleen & & $251(45.4)$ & $16(28.6)$ & $235(47.3)$ & 0.0077 \\
\hline Kidney & & $102(18.4)$ & $12(21.4)$ & $90(18.1)$ & 0.5437 \\
\hline Adrenal & & $64(11.6)$ & $4(7.1)$ & $60(12)$ & 0.2762 \\
\hline Aortocaval vessels & & $14(2.5)$ & $3(5.4)$ & $11(2.2)$ & $0.1605^{b}$ \\
\hline Pancreas & & $13(2.3)$ & $3(5.4)$ & $10(2)$ & $0.1349^{b}$ \\
\hline Bladder & & $4(0.7)$ & $2(3.6)$ & $2(0.4)$ & $0.0527^{b}$ \\
\hline Abdominal anterior wall & & $132(23.7)$ & $34(60.7)$ & $98(19.6)$ & $<0.0001$ \\
\hline Lumbar spine & & $146(26.3)$ & $11(19.6)$ & $135(27)$ & 0.2354 \\
\hline Pelvic ring & & $151(27.2)$ & $12(21.4)$ & $139(27.8)$ & 0.3094 \\
\hline
\end{tabular}

a 2 missing data because of no abdominal contrast due to heart failure

${ }^{\mathrm{b}}$ Fisher tests, others are chi-square tests

(PPV), $99.6 \%$ negative predictive value (NPV), 11.4 positive likelihood ratio (PLR) and 0.04 negative likelihood ratio (NLR) in our cohort.

The internal bootstrap validation gave the following $95 \%$ confidence intervals (CI) for the performance of our scoring system: sensitivity,91.7-100\%; specificity, 85.7-97.6 \%; PPV, 41.4-82.3 \%; NPV, 98.9-100\%; and AUC, 97.5-99.3\%.

\section{Reproducibility}

Interobserver agreement is reported in Table 3. Interobserver agreement was substantial to almost perfect for $6 / 7$ direct CT signs of BBMI which belonged to our $\mathrm{CT}$ score, and moderate for the bowel wall thickening sign $(\kappa=0.60)$. It was almost perfect for both detection of anterior abdominal wall injury $(\kappa=0.97$, CI 0.94-1) and splenic injury $(K=1)$.

\section{Discussion}

We developed a reliable scoring system which can be easily implemented and could allow surgeons to optimize decisions to operate on multi-trauma patients. To our knowledge, this is the largest reported cohort of BBMI requiring surgery with CT in blunt trauma $(n=56)$, which compares favourably with the findings of the studies by Atri et al. [9], Steenburg et al. [20] and $\mathrm{Wu}$ et al. [15], including 38, 15 and 13 patients with surgical BBMI, respectively. Moreover, this is the largest consecutive cohort of abdominal trauma cases with $\mathrm{CT}$ that has been entirely screened to assess the predictive value of $\mathrm{CT}$ findings for surgical BBMI.

Risk scoring systems are widely used in trauma centres and ideally have the triple advantage of predicting patient outcome, facilitating decision making and enabling comparisons when benchmarking the performance of clinical units. They 
Table 3 Diagnostic performance and interobserver reproducibility of each CT finding

\begin{tabular}{|c|c|c|c|c|c|c|c|}
\hline & & Sensitivity & Specificity & PPV & NPV & kappa & $95 \%$ CI kappa \\
\hline \multirow[t]{3}{*}{ Haemoperitoneum } & & & & & & 0.79 & $0.72-0.87$ \\
\hline & 1 (small amount) & $25(14-36)$ & $56.2(52-61)$ & $6(3-9)$ & $87(83-91)$ & & \\
\hline & 2 (abundant) & $69.6(58-82)$ & $74.6(70-78)$ & $23.5(17-31)$ & $95.6(94-98)$ & & \\
\hline Free pneumoperitoneum & & $30.4(18-42)$ & $99.2(98-100)$ & $81(64-98)$ & $92.7(91-95)$ & 0.89 & $0.77-1$ \\
\hline Mesenteric pneumoperitoneum & & $17.9(8-28)$ & $99.6(99-100)$ & $83(68-100)$ & $91.5(89-94)$ & 0.72 & $0.53-0.92$ \\
\hline Bowel wall thickness & & $67.9(56-80)$ & $91.2(89-94)$ & $46.3(36-57)$ & $96.2(95-98)$ & 0.60 & $0.48-0.70$ \\
\hline \multirow[t]{3}{*}{ Bowel wall thickening length } & & & & & & 0.61 & $0.50-0.72$ \\
\hline & Focal & $35.7(23-48)$ & $94.2(92-96)$ & $40.8(27-55)$ & $92.9(91-95)$ & & \\
\hline & Non-focal & $32.1(20-44)$ & $97(95-98)$ & $54.5(36-72)$ & $92.7(90-95)$ & & \\
\hline Arterial mesenteric vessel extravasation & & $26.8(15-38)$ & $98.8(98-100)$ & $71.4(53-90)$ & $92.3(90-95)$ & 0.90 & $0.78-1$ \\
\hline Mesenteric haematoma & & $42.9(30-56)$ & $94(91-96)$ & $42.9(30-56)$ & $93.6(91-96)$ & 0.75 & $0.62-0.88$ \\
\hline Mesenteric stranding & & $58.9(46-72)$ & $89.6(87-92)$ & $38.8(28-49)$ & $95.1(93-97)$ & 0.64 & $0.53-0.75$ \\
\hline Abrupt termination of mesenteric vessel & & $25(14-36)$ & $99.2(98-100)$ & $77.8(52-94)$ & $92.1(90-94)$ & 0.57 & $0.28-0.86$ \\
\hline Beaded mesenteric vessel & & $12(4-21)$ & $98.8(98-100)$ & $53.8(27-81)$ & $90.9(89-93)$ & 0.59 & $0.37-0.81$ \\
\hline Decreased bowel wall enhancement & & $39.3(26-52)$ & $97.8(96-99)$ & $66.7(51-83)$ & $93.5(91-96)$ & 0.68 & $0.50-0.85$ \\
\hline Bowel wall discontinuity & & $33.9(21-46)$ & $99.8(99-100)$ & $95(85-100)$ & $93.1(91-95)$ & 0.75 & $0.58-0.91$ \\
\hline
\end{tabular}

Data are percentages and numbers in brackets are $95 \%$ confidence intervals

must be developed using simple, reliable and reproducible parameters. The CT scoring system we developed fulfils the requirements and goals of trauma scoring systems. It may facilitate the selection of patients requiring abdominal surgical management according to a standardized $\mathrm{CT}$ analysis with reliable prediction of surgical BBMI (bootstrap AUC $=97.5-$ $99.3 \%$ ). Our scoring system is easy to use, based on a thorough CT reading with excellent reproducibility (substantial or almost perfect agreement for 8/9 CT signs), and achieves very high predictive values with an 11-fold increased pretest probability of surgical BBMI if the score is $\geq 5$ (PLR 11.4), and a high negative predictive value $(99.6 \% ; 95 \%$ CI bootstrap 98.9-100 \%) if the score is $<5$. This is of paramount importance because the most common missed injuries in the trauma imaging era are in the bowel region [12], and misdiagnosis of surgical BBMI could delay appropriate management and often results in significant morbidity and mortality. While maintaining this high NPV level, we obtained a PPV ranging from 41.4 to $82.3 \%$ as estimated by the bootstrap calculation, thus limiting the number of non-therapeutic laparotomies.

In fact, two CT signs (mesenteric pneumoperitoneum and bowel wall discontinuity) were sufficient to confirm the presence of surgical BBMI, with a score equal to 5 (Figs. 3 and 4). Mesenteric pneumoperitoneum was highly associated with surgical BBMI (OR 140.5, 95 \% CI 9.3->999.9). Although free pneumoperitoneum also has very good reliability for the diagnosis of traumatic perforation, it does not appear significant in our multivariate analysis, as there was a statistical

Table 4 Significant CT signs in multivariate analysis to predict the risk of surgical BBMI and their assigned numerical CT score according to the logistic regression estimates ( $\beta$ parameter)

\begin{tabular}{|c|c|c|c|c|c|c|}
\hline & CT signs & $p$ value & Odds ratio & $95 \% \mathrm{CI}$ odds ratio & $\beta$ estimate & Score \\
\hline \multirow[t]{3}{*}{1} & Haemoperitoneum & 0.0017 & & & & \\
\hline & Small amount & & 3.3 & $0.4-23.8$ & 1.1944 & 1 \\
\hline & Abundant & & 16.3 & $2.4-111.4$ & 2.7904 & 3 \\
\hline 2 & Mesenteric pneumoperitoneum & 0.0003 & 140.5 & $9.3->999.9$ & 4.9456 & 5 \\
\hline 3 & Bowel wall thickness & 0.0001 & 9.8 & $3.1-31.4$ & 2.2878 & 2 \\
\hline 4 & Arterial mesenteric vessel extravasation & 0.0002 & 16.8 & $3.7-75.7$ & 2.8225 & 3 \\
\hline 5 & Mesenteric stranding & 0.0019 & 5.2 & $1.8-14.9$ & 1.6541 & 2 \\
\hline 6 & Reduced bowel wall enhancement & 0.0306 & 4.4 & $1.2-17.0$ & 1.4856 & 1 \\
\hline 7 & Bowel wall discontinuity & 0.0003 & 128.9 & $9.5->999.9$ & 4.8593 & 5 \\
\hline 8 & Splenic injury & 0.0467 & 0.3 & $0.1-0.9$ & -1.1123 & -1 \\
\hline 9 & Anterior abdominal wall injury & 0.0002 & 8.3 & $2.7-25.2$ & 2.1188 & 2 \\
\hline
\end{tabular}



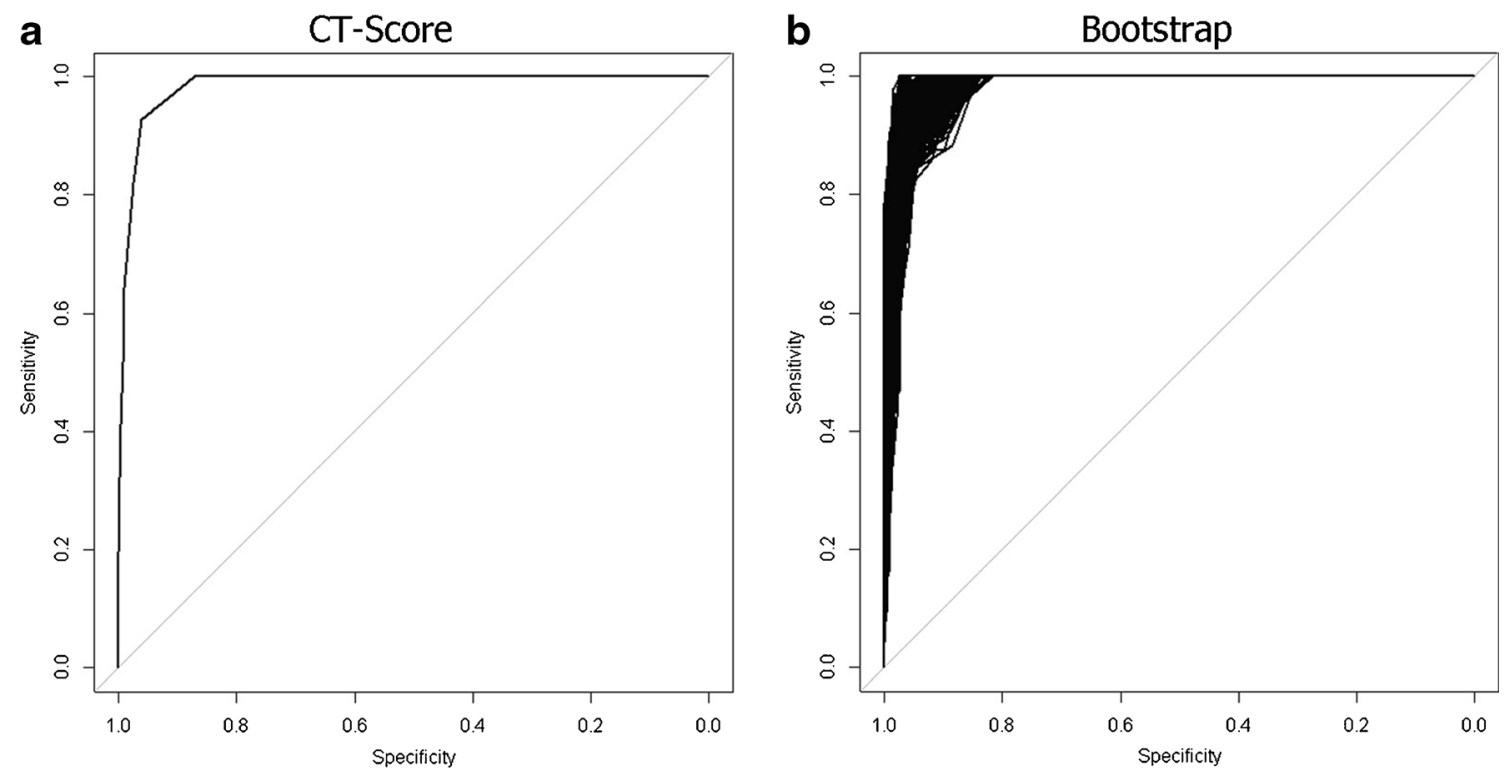

Fig.2 ROC curve built from our scoring system (a), and ROC curve derived from the bootstrap analysis (b)

relationship between free and mesenteric pneumoperitoneum. There are classical causes of free pneumoperitoneum which are not due to bowel wall trauma, such as rupture of bladder with Foley catheter in place or diffusion of a pneumothorax in patients with diaphragmatic rupture [5]. Furthermore, in traumatic bowel perforation, mesenteric pneumoperitoneum may be useful for localizing the damaged intestinal tract occurring more commonly in the small bowel [21]. In contrast, mesenteric air is more commonly missing than free pneumoperitoneum in bowel perforation (these two findings were lacking in $29.6 \%$ of bowel perforations in our study), as already reported in the literature $[8,21]$.

Visualization of a bowel wall discontinuity had the best positive predictive values of surgical BBMI (PPV=95\%)
Fig.3 Axial abdominal contrast material-enhanced CT image acquired at arterial (a), venous (b, d) and delayed (c) phases, in a 35year-old man after motor vehicle crash. Note the active mesenteric vessel extravasation appearing in the arterial phase and expanded at the later phases (arrow), and the bowel wall discontinuity in an ileal loop (arrowhead) with free pneumoperitoneum (asterisk). Findings at laparotomy included an ileal perforation requiring suture, and an inferior mesenteric artery rupture requiring haemostasis and left hemicolectomy for ischemia
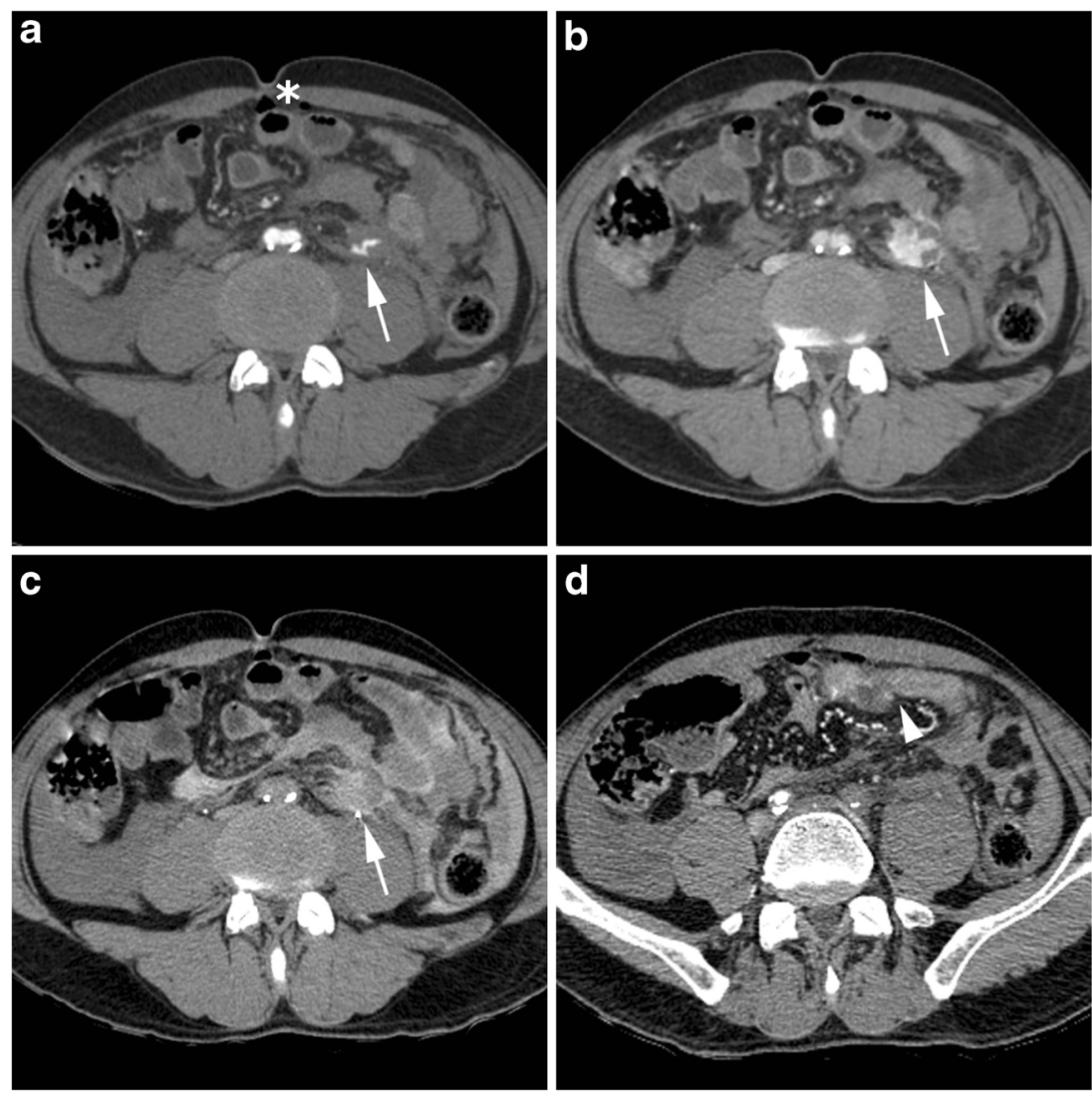


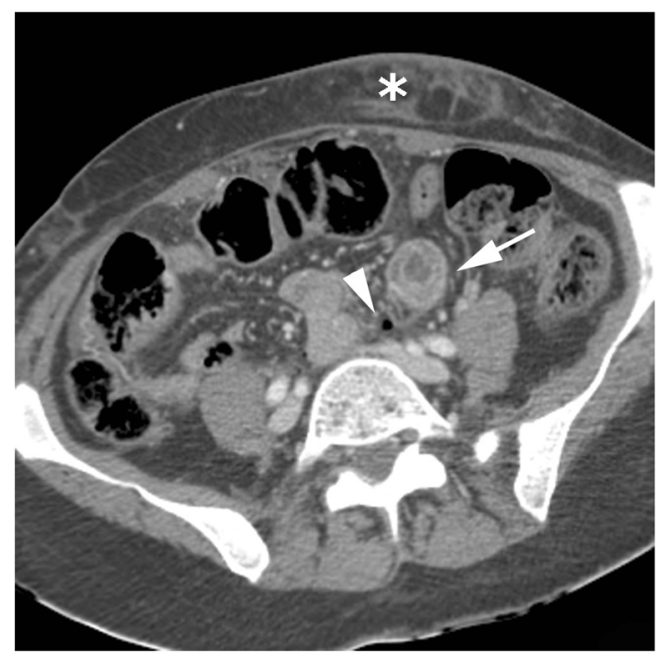

Fig.4 Axial abdominal contrast-enhanced CT image acquired at venous phase in a 59-year-old woman after motor vehicle crash. Note the mesenteric pneumoperitoneum (arrowhead) close to a focal small bowel wall thickening (arrow) and with subcutaneous fat stranding along the course of the fastened seat belt (asterisk). Findings at laparotomy included a 2$\mathrm{mm}$ jejunal perforation and a transverse colic perforation, all requiring suture

among all CT signs studied, as already reported [20]. This is a direct CT sign of a perforated loop.

Arterial mesenteric extravasation had an odds ratio of 16.8 (95\% CI 3.7-75.7). The logistic regression analysis assigned a value of 3 , which is inferior to the cut-off of 5 determined by our ROC curve analysis. This could be surprising since it is well established that active mesenteric vessel extravasation is a finding of BBMI requiring surgical exploration both for stopping the bleeding and for investigating the bowel because of the risk of ischemia. However mesenteric vessel extravasation is always associated with at least mesenteric stranding if not an abundant haemoperitoneum. Hence, the combination of both signs results in a score of at least 5 , which is predictive of surgical BBMI.

Mesenteric haematoma and mesenteric stranding had very limited reliability for diagnosing surgical BBMI. These two findings had a positive predictive value for the diagnosis of surgical BBMI inferior to $50 \%$. This confirms that although a mesenteric finding is suggestive and mesenteric haematoma is specific of mesenteric injury, these signs do not always indicate a need for surgery [5].

Although we did not consider diffuse thickening of the bowel with other CT findings of shock as a finding of bowel trauma, bowel wall thickness had a positive predictive value for surgical BBMI inferior to $50 \%$ and was assigned a score of 2, insufficient in itself to predict a surgical BBMI. Different criteria have been used in the literature to diagnose small bowel or colon wall thickening [22]. By using the same criteria to define bowel wall thickening as used in our study, Atri et al. [9] have already shown that bowel wall thickening may be present despite the lack of important bowel injury requiring surgery. Numerous causes may explain bowel thickening in patients without surgical BBMI: non-significant bowel injuries such as a haematoma or a tear limited to the serosa [5], collapsed bowel lumen, or normal variants [22, 23].

Areas of decreased or absent bowel wall contrast enhancement, which is classically indicative of ischemic bowel wall in abdominal emergencies [24], were significantly associated with surgical BBMI (OR 4.4, 95 \% CI 1.2-17). However, there were 11 false positives $(33.3 \%)$ in our study. This could be explained by the difficulty of analysing bowel wall enhancement in multiple trauma patients with heart failure, abundant haemoperitoneum and sometimes hypoperfusion complex [25], because in such cases mucosal bowel enhancement is often intense and diffuse, which makes comparisons tricky with the seemingly less enhanced normal adjacent enhanced bowel.

We note an association between surgical BBMI and abdominal wall injuries. This highlights one of the well-known mechanisms of BBMI due to direct impact [26] and anterior compression of the abdomen classically caused by a fastened seatbelt, whereby the bowel loop may be crushed between the seatbelt and the lumbar spine, leading to bowel perforation. We think that abdominal anterior injury visualized in $\mathrm{CT}$ examination is helpful for predicting surgical BBMI, when combined with other $\mathrm{CT}$ score findings. By contrast, we report an inverse association between spleen lesion and surgical BBMI. Although the weight of this inverse association is weak (assigned score of -1 ), we think that in patients with an abundant haemoperitoneum (score of 3 ), the presence of a spleen lesion decreases the risk of surgical BBMI, probably because haemoperitoneum is explained by splenic injury and not by a mesenteric injury.

Our study had some limitations. First, it was a retrospective study with its inherent bias and was conducted in a single centre, but it included consecutive patients with blunt abdominal trauma investigated by CT. Secondly, there was a higher prevalence of surgical BBMI in our study (10.1\%) than reported in previous studies, i.e. twofold lower [7, 17]. This was due to patient selection bias, as patients had more serious injuries in this study (average ISS 14) and were selected on the basis of an abdominal trauma that did not reflect the entire trauma population. The prevalence in our study is actually close to that documented in other cohorts of blunt abdominal trauma patients, with a reported prevalence of surgical BBMI of $12.3 \%$ in the study by $\mathrm{Wu}$ et al. [15], and $15.3 \%$ in the study by Tan et al. [14]. Interestingly, in our study, surgical BBMI constituted the majority of blunt abdominal trauma patients explored by laparotomy, and currently represents the main reason for laparotomy [27]. Thirdly, although the internal validation of our score indicated high diagnostic performance, an external validation set is essential to confirm the diagnostic value before generalization. 
In conclusion, we developed an easy to implement and reliable scoring system based on CT findings, which is highly predictive of surgical BBMI. When the CT score is at least 5 , then a surgical procedure should be carried out given the high probability of BBMI requiring surgical repair. Conversely, when the CT score is less than 5, then non-operative management should be prescribed along with clinical follow-up and possibly repetition of abdominal CT examination within 8-12 h.

Acknowledgements The scientific guarantor of this publication is Prof. Taourel. The authors of this manuscript declare no relationships with any companies whose products or services may be related to the subject matter of the article. The authors state that this work has not received any funding. One of the authors has significant statistical expertise. Institutional review board approval was obtained. Written informed consent was waived by the institutional review board. Methodology: retrospective, diagnostic or prognostic study, performed at one institution.

\section{References}

1. Watts DD, Fakhry SM (2003) Incidence of hollow viscus injury in blunt trauma: an analysis from 275,557 trauma admissions from the East multi-institutional trial. J Trauma 54(2):289-294

2. Killeen KL, Shanmuganathan K, Poletti PA, Cooper C, Mirvis SE (2001) Helical computed tomography of bowel and mesenteric injuries. J Trauma 51(1):26-36

3. Scaglione M, de Lutio di Castelguidone E, Scialpi M, Merola S, Diettrich AI, Lombardo P et al (2004) Blunt trauma to the gastrointestinal tract and mesentery: is there a role for helical CT in the decision-making process? Eur J Radiol 50(1):67-73

4. Frellesen C, Stock W, Kerl JM, Lehnert T, Wichmann JL, Nau C et al (2014) Topogram-based automated selection of the tube potential and current in thoraco-abdominal trauma CT - a comparison to fixed $\mathrm{kV}$ with mAs modulation alone. Eur Radiol 24(7):17251734

5. Brofman N, Atri M, Hanson JM, Grinblat L, Chughtai T, Brenneman F (2006) Evaluation of bowel and mesenteric blunt trauma with multidetector CT. Radiographics 26(4):1119-1131

6. Janzen DL, Zwirewich CV, Breen DJ, Nagy A (1998) Diagnostic accuracy of helical CT for detection of blunt bowel and mesenteric injuries. Clin Radiol 53(3):193-197

7. Malhotra AK, Fabian TC, Katsis SB, Gavant ML, Croce MA (2000) Blunt bowel and mesenteric injuries: the role of screening computed tomography. J Trauma 48(6):991-998, discussion 9981000

8. Mirvis SE, Gens DR, Shanmuganathan K (1992) Rupture of the bowel after blunt abdominal trauma: diagnosis with CT. AJR Am J Roentgenol 159(6):1217-1221

9. Atri M, Hanson JM, Grinblat L, Brofman N, Chughtai T, Tomlinson G (2008) Surgically important bowel and/or mesenteric injury in blunt trauma: accuracy of multidetector CT for evaluation. Radiology 249(2):524-533

10. Ekeh AP, Saxe J, Walusimbi M, Tchorz KM, Woods RJ, Anderson HL et al (2008) Diagnosis of blunt intestinal and mesenteric injury in the era of multidetector CT technology-are results better? J Trauma 65(2):354-359

11. Elton C, Riaz AA, Young N, Schamschula R, Papadopoulos B, Malka V (2005) Accuracy of computed tomography in the detection of blunt bowel and mesenteric injuries. Br J Surg 92(8):10241028

12. Matsushima K, Mangel PS, Schaefer EW, Frankel HL (2013) Blunt hollow viscus and mesenteric injury: still underrecognized. World $\mathrm{J}$ Surg 37(4):759-765

13. Petrosoniak A, Engels PT, Hamilton P, Tien HC (2013) Detection of significant bowel and mesenteric injuries in blunt abdominal trauma with 64-slice computed tomography. J Trauma Acute Care Surg 74(4):1081-1086

14. Tan K-K, Liu JZ, Go T-S, Vijayan A, Chiu M-T (2010) Computed tomography has an important role in hollow viscus and mesenteric injuries after blunt abdominal trauma. Injury 41(5):475-478

15. Wu C-H, Wang L-J, Wong Y-C, Fang J-F, Lin B-C, Chen H-W et al (2011) Contrast-enhanced multiphasic computed tomography for identifying life-threatening mesenteric hemorrhage and transmural bowel injuries. J Trauma 71(3):543-548

16. Federle MP, Jeffrey RB (1983) Hemoperitoneum studied by computed tomography. Radiology 148(1):187-192

17. Wisner DH, Chun Y, Blaisdell FW (1990) Blunt intestinal injury. Keys to diagnosis and management. Arch Surg 125(10):13191322, discussion 1322-1323

18. Murakami AM, Anderson SW, Soto JA, Kertesz JL, Ozonoff A, Rhea JT (2009) Active extravasation of the abdomen and pelvis in trauma using 64MDCT. Emerg Radiol 16(5):375-382

19. Shanmuganathan K, Mirvis SE, Sover ER (1993) Value of contrastenhanced CT in detecting active hemorrhage in patients with blunt abdominal or pelvic trauma. AJR Am J Roentgenol 161(1):65-69

20. Steenburg SD, Petersen MJ, Shen C, Lin H (2014) Multi-detector $\mathrm{CT}$ of blunt mesenteric injuries: usefulness of imaging findings for predicting surgically significant bowel injuries. Abdom Imaging. doi:10.1007/s00261-014-0262-2

21. Kim HC, Shin HC, Park SJ, Park SI, Kim HH, Bae WK et al (2004) Traumatic bowel perforation: analysis of CT findings according to the perforation site and the elapsed time since accident. Clin Imaging 28(5):334-339

22. Macari M, Balthazar EJ (2001) CT of bowel wall thickening: significance and pitfalls of interpretation. AJR Am J Roentgenol 176(5):1105-1116

23. Wittenberg J, Harisinghani MG, Jhaveri K, Varghese J, Mueller PR (2002) Algorithmic approach to CT diagnosis of the abnormal bowel wall. Radiographics 22(5):1093-1107

24. Geffroy Y, Boulay-Coletta I, Jullès M-C, Nakache S, Taourel P, Zins $M$ (2014) Increased unenhanced bowel-wall attenuation at multidetector CT is highly specific of ischemia complicating small-bowel obstruction. Radiology 270(1):159-167

25. Ames JT, Federle MP (2009) CT hypotension complex (shock bowel) is not always due to traumatic hypovolemic shock. AJR Am J Roentgenol 192(5):W230-W235

26. Hughes TMD, Elton C (2002) The pathophysiology and management of bowel and mesenteric injuries due to blunt trauma. Injury 33(4):295-302

27. Sorensen VJ, Mikhail JN, Karmy-Jones RC (2002) Is delayed laparotomy for blunt abdominal trauma a valid quality improvement measure in the era of nonoperative management of abdominal injuries? J Trauma 52(3):426-433 